\title{
Temporal Structure of Discourse*
}

\author{
Irene Pimenta Rodrigues \\ José Gabriel P. Lopes \\ Centro de Inteligência Artificial, UNINOVA \\ Quinta da Torre, 2825 Monte da Caparica Portugal \\ email:(ipr,gpl)@fct.unl.pt
}

\begin{abstract}
In this paper discourse segments are defined and a method for discourse segmentation primarily based on abduction of temporal relations between segments is proposed. This method is precise and computationally feasible and is supported by previous work in the area of temporal anaphora resolution.
\end{abstract}

\section{Introduction}

B. Webber in [22] explains how discourse segmentation contributes to the interpretation of tense. In this paper we discuss how "tense interpretation" contributes to discourse segmentation. Following Webber's operational definition of segments [21] we present the data structures necessary for representing discourse segments and an algorithm to perform discourse segmentation.

In order to build a discourse segment structure some clear criteria for grouping chunks of text into segments and for grouping these segments into other segments must be available. Our criterion is based on the maintenance of temporal coherence between segments. It relies on the abduction of temporal relations between segments that necessarily have temporal properties.

Abduction of temporal relations between segments is a feasible and precise method for discourse segmentation. This is the leading criterion for segmentation and does not prevent us from using other criteria such as clue words, spatial relations and recognition of state elaboration. Current discourse structure theories use criteria such as rhetorical relations $[9,15,13]$, intention recognition [7], narrative discontinuities [18], etc. All of them use a temporal criterion for segmentation embedded in less manageable

This work has been supported by JNICT, INIC and Gabinete de Filosofia do Conhecimento. criteria such as "the increasing desire of $\mathbf{R}$ to perform action $\mathrm{N}^{\mathrm{n}}$.

Our discourse segmentation is accomplished in order to enable us to address the following discourse phenomena:

- Temporal anaphora - the interpretation of tense and aspect depends on the discourse structure [22]. The maintenance of a "Temporal Focus" is suggested by some authors $[11,22,18,5]$ for tense interpretation. Based on their work our main concern is to provide the "temporal focus" for tense interpretation. In our segment structure the temporal focus is the set of visible segments. A pop in the temporal focus structure will imply the closing of one or more segments.

- This and that anaphora - These pronouns may refer to discourse segments [21]. With our segmentation we provide discourse segments for reference.

- Pronominal and definite anaphora - the interpretation of pronouns and definite nominals depends on the attentional structure (Grosz and Sidner). The attentional structure depends on the discourse segmentation. With our approach to segmentation the attentional structure can be computed from our discourse structure - our visible segments provide visible entities and sequents for these anaphors.

- event reference - our approach provides a representation for eventualities, the discourse referents and the attentional structure necessary for solving this kind of anaphora.

- temporal coherence - is achieved by inferring one of the possible temporal relations between two eventualities [14]. Our segmentation process mainly controlled by abduction of temporal relations between eventualities enables us to check if a text is temporally coherent. Moreover as we propagate temporal constraints through discourse structure the number of even-

\footnotetext{
${ }^{1}$ Definition of the rhetorical relation motivation [15].
} 
tualities that must be temporally related with a new eventuality increases.

- temporal reliability - is achieved by the existence of a model for the temporal relations inferred. During discourse processing we build a temporal structure where all the temporal constraints can be checked. This structure is updated when a new temporal referent or a newly abducted temporal relation is added. Thus temporal reliability is granted.

- discourse coherence - is difficult to check by using only our discourse structure. It requires more processing, namely the ability to find intentions for the segments.

Tense interpretation contributes to segmentation by defining the temporal relation between the segment used as reference and the segment that represents the tensed sentence to be interpreted. Thus tense interpretation allows the choice of a segment by indicating where to attach the new sentence segment and greatly restricts the possible referents for anaphora res olution. A failure in satisfying structural constraints results in the choice of another segment referent.

The temporal anchoring of eventualities assumes that there is some temporal representation for the eventualities. In this paper we use a representation for eventualities close to the event calculus[12], and a graph structure for time representation. Updating the discourse structure will be equivalent to updating a temporal data base. Discourse referents are existentially quantified variables that can be further constrained by the analysis of a new discourse sentence.

In the following sections the temporal relations used in this paper are defined, followed by an explanation of our notion of segments, their properties and the algorithm for discourse segmentation. A detailed example will be worked out. Finally a conclusion and a comparison of our work with related work in this area is presented.

\section{Temporal relations}

The semantic representation of an eventuality includes a time interval where the eventuality must be true so that the sentence and the discourse can be true. The time interval will be represented by two time points. Temporal relations between two time intervals can be expressed by relations between the extremes of the time intervals. So instead of using the 13 rela- tions proposed by Allen [1] we have chosen to use just 5 relations.

- $t_{a_{1}}<t_{e_{2}} \equiv t_{1_{1}}<t_{e_{3_{i}}}$ - this relation is like Allen's relation before or meets.

- $t_{a_{1}}>t_{s_{2}} \equiv t_{a_{2}}<t_{a_{1}}$ - this relation is like Allen's relation after or met-by.

- $t_{s_{1}} \subset t_{s_{2}} \equiv t_{s_{i}}<t_{n_{1},}, t_{n_{1},}<t_{n_{j}}$ - this relation jo like Allen's relation $I N$.

- $t_{a_{1}} \supset t_{o_{2}} \equiv t_{a_{1}}<t_{a_{i}}, t_{a_{3}}<t_{s_{1}}$ - this relation is like Allen's relation $O N$.

$\bullet t_{a_{1}} \times t_{a_{3}} \equiv \exists t: t \subset t_{a_{1}}, t \subset t_{a_{2}}$ - this relation is like Allen's relation!.

These 5 relations are enough for translating natural language sentences as it is difficult to express the 13 relations of Allen and their $2^{13}$ combinations in natural language. F. van Eynde [6] presents the set of relations necessary for the temporal systems he studied (for EEC languages). Our relation set, however, is smaller. As sentence aspect coerces the verb aspectual class to change [16], v. Eynde's overlap relations may be rewritten using the relations $<$ and $>$.

The following examples will demonstrate our use of the temporal relations. For the sake of simplicity only discourse referents introduced by eventualities and time intervals are represented. Eventualities introduced by nominals (as in example I "his key") are discarded.

I) John picked off his key(1). He opened the door(2).

The eventualities of sentences (1) and (2) are: event $\left(e_{1}, \operatorname{pick}(j o h n, \operatorname{key} 1)\right)$, time $\left(e_{1}, t_{a_{1}}\right)$; event $\left(e_{2}, \operatorname{open}(j o h n\right.$, door 1$\left.)\right)$, time $\left(e_{2}, t_{2,2}\right)$; the temporal relation is $t_{t_{1}}<t_{s_{3}}$.

II) John bought an umbrella(1). He had lost his umbrella(2).

The eventualities of sentences (1) and (2) are: event $\left(e_{1}, b u y(j o h n, u m b 1)\right)$, time $\left(e_{1}, t_{s_{1}}\right)$ event $\left(e_{2}\right.$, loose $\left.(j o h n, u m b 2)\right)$, time $\left(e_{2}, t_{n_{2}}\right)$; the temporal relation is $t_{s_{1}}>t_{t_{2}}$.

III) John bought an umbrella (1). It was raining(2).

The eventualities of sentence (1) and (2) are: event $\left(e_{1}\right.$, buy $\left.(j o h n, u m b 1)\right), \operatorname{time}\left(e_{1}, l_{s_{1}}\right)$; event $\left(e_{2}\right.$, rain $)$, time $\left(e_{2}, t_{0,}\right)$;

the temporal relation is $t_{1_{1}} \subset t_{n_{2}}$.

IV) John had a nice meal(1). He ate salmon(2). The eventualities of sentence (1) and (2) are: event $\left(e_{1}\right.$, have $(j o h n$, meal $\left.)\right)$, time $\left(e_{1}, t_{a_{1}}\right)$; event $\left(e_{2}\right.$, eat(john,salmon)), time $\left(e_{2}, t_{t_{2}}\right)$; the temporal relation is $t_{t_{2}} \supset t_{0_{2}}$.

V) It was raining(1). There was a strong wind(2). 
The eventualities of sentence (1) and (2) are: event $\left(e_{1}, \operatorname{rain}\right)$, time $\left(e_{1}, t_{n_{1}}\right)$;

event $\left(e_{2}\right.$, wind_strong), time $\left(e_{2}, t_{t_{3}}\right)$;

the temporal relation is $t_{s_{1}} \propto t_{t_{2}}$.

VI) John sat down on a chair(1). Mary lied down on a sofa(2).

The eventualities of sentence (1) and (2) are: event $\left(e_{1}\right.$, sit (john, chairl $\left.)\right)$, time $\left(e_{1}, t_{s_{1}}\right)$; event ( $e_{2}, \operatorname{lie}($ Mary, sofa $\left.)\right)$, time $\left(e_{2}, t_{o_{2}}\right)$; the temporal relation is $t_{s_{1}}$ none $t_{n_{2}}$ as these two eventualities are independent.

\section{Discourse Segments}

A discourse segment is a discourse object. It is represented by a discourse referent that can be used for later reference. In contrast to other discourse theories, segments as dynamic structures that help to define context interpretations are considered as real discourse objects. Thus in our approach we use segments as objects with properties that will be defined later. A text is represented by a segment and a segment supplies context information for the semantic interpretation during discourse processing.

Next segments will be defined as well as their construction and use in the semantic interpretation.

\subsection{Kind of segments}

We distinguish two kinds of segments: basic and non-basic ones.

A basic segment represents an eventuality plus some features, typically syntactic ones like tense and aspect (the leaves of fig. 1).

A nonbasic segment has one or more subseg ments (basic or not) obeying to a set of temporal constraints and a set of features. Every nonbasic segment has a sort depending on the temporal constraints it imposes on its subsegments.

Segment features are necessary for discourse reasoning. Some of them may be dropped after a closing but others have to remain until the discourse is completely processed. The features we take into account in this paper are the following

- tense - The feature tense is needed for temporal anaphora resolution.

- eventuality - The semantic representation of an eventuality is important for temporal anaphora resolution, for causal reasoning and other kinds of reasoning that depend on the kind of the eventuality.

- eventuality time - This is the main issue in the definition of a segment as the abducted relation between eventuality times determines the segment structure's behavior.

- discourse referents - for solving discourse reference.

- subsegments - an ordered list containing all its subsegments.

\subsection{Sorts of segments}

Depending on the abducted temporal relation between eventualities in a discourse, the eventualities are grouped into different sorts of segments. Using the above mentioned five temporal relations seven sorts of segments ${ }^{2}$ can be defined, e.g.

1. basic - the minimal segment.

2. none - this segment does not impose any restriction on the temporal relation of its subsegments. The discourse of example VI will be represented by this sort of segment.

3. sequence - the subsegments in the list of segments are temporally ordered, e.g. ex I (fig. 1.a).

4. tb - contains only two subsegments with the first one temporally situated after the second one, e.g. ex II (fig. 1.b).

5. bk - has two subsegments with the first one temporally contained in the second one, e.g.ex III.

6. elab - has two subsegments with the first one temporally containing the second one, e.g. ex IV.

7. over - every segment in the list of subsegments must temporally intersect a nonempty time interval, e.g. ex V.

For each sort of segment it must be defined how to compute its features representing properties from the features of its subsegments.

\subsection{Properties of Segments}

Segments that can have a list of subsegments containing an unlimited number of segments are none, sequence and over. These segments can be augmented during discourse processing. The features of these segments are the following:

- none - The feature eventuality contains the set of all subsegments' eventualities, while the

\footnotetext{
2 The names of these ecgments are abbrevintions of some rhetorical relations that impose the ane temporal constraints. These abrevintions should not be read as if they meant the same as the rhetorical relations. They just mean that their subsegments obey a particular temporal relation.
} 


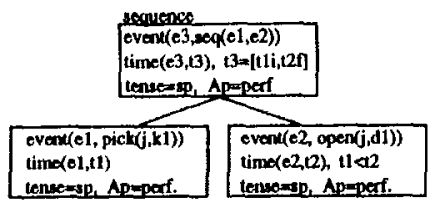

(a)

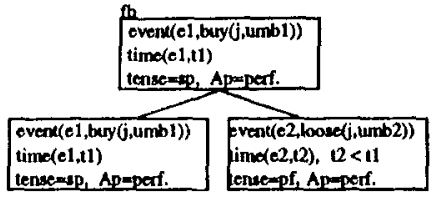

(b)

Figure 1: Segmentation for ex. I and II

feature time contains the set of all subsegments' times. The tense feature refers to the tense of the last subsegment.

- sequence - the feature eventuality is the composition of all the segments' eventualities obtained by the seq operator. The time feature interval is the time interval $\left[t_{s_{i}}, t_{n_{j}}\right]$, where $t_{1_{i}}$ is the initial point of the first segment time inter$\mathrm{val}$ and $t_{n_{n},}$ is the final point of the last segment time interval. The tense feature is the tense of the last segment (fig. 1.a). This segment can be augmented by adding a new segment to the list of segments. In this case the features of the seguence segment have to be evaluated again ${ }^{3}$.

- over - the feature eventuality is unknown, the feature time is the time interval of the intersection of all time segments. The tense feature is the tense of its last subsegment.

The segments that have two subsegments are $f b, b k$, and elab. These segments can only be augmented by the replacement of its second subsegment by a new one obeying the same set of constraints. 'The replaced segment is the first subsegment of the new one. These segments have the same features of the first subsegment (fig. 1.b for a segment of sort $f b$ ).

\subsection{Discourse Segmentation}

Discourse segmentation will be done incrementally. A sentence will be represented by a segment. The processed discourse will be represented by a segment.

\footnotetext{
${ }^{3}$ A notion similar to the acquence wegment is used in other theories for discourse segmentation named in RST [15] a" "narration", in Hobbe [9] also as "narration" and in planning $[10]$ an "sequence".
}

The steps for the discourse segmentation are:

1. to obtain the first sentence segment. This segment will be the discourse segment.

2. to obtain the segment representing the next discourse sentence.

3. to insert the new segment in the discourse segment. This step will have the following substeps:

(a) To compute the set of visible segments (i.e. the right subsegments of the discourse segment) from the discourse segment. This set of segments can be ordered by some discourse preference rule, e.g. we may prefer to continue the current segment or prefer to continue the first opened segment and close the other ones ${ }^{4}$.

(b) to choose one segment $s_{1}$ from the set of visible segments.

(c) to add the new sentence segment $s_{2}$ to segment $s_{l}$ by:

- continuing the subsegment list of $s_{1}$ if $s_{2}$ can satisfy the constraints of $s_{1}$.

- substituting $s_{1}$ by a new one $s_{3} . s_{3}$ contains $s_{1}$ as first subsegment and $s_{2}$ as second subsegment in its subsegment list. The sort of segment $8_{3}$ is one of the 6 nonbasic ones.

(d) if it is not possible to add the new segment then choose another segment from the set of the visible ones, call it $s_{1}$ and try again going back to step 3c.

4. go back to step 2 if there are more sentences to process in the discourse.

\subsection{Abduction of temporal relations}

The main process in discourse segmentation is to check for temporal relations between segments because this is the only criterion used for segmentation.

For deciding how to link segment $B_{2}$ given segment $s_{1}$, do:

1. find the referent for $s_{2}$.

- if $s_{1}$ is going to be continued by $s_{2}$, then the referent will be the last subsegment in the subsegment list of segment $s_{1}$.

- if $s_{1}$ is going to be substituted by a new segment $s_{3}$ then the referent will be $s_{1}$.

\footnotetext{
The way segments are ordered will have consequences on the discourse segmentation. In cases where there are more then one possible segmentation preference will be given to the first one.
} 
2. After obtaining a referent, abduct the temporal relation between $s_{2}$ and the referent.

3. After getting the relation between $s_{2}$ and the referent, do:

- if $s_{1}$ is to be continued by $s_{2}$ then check if $s_{2}$ satisfies the set of constraints of $s_{1}$. If so, update the $s_{1}$ features if needed and repeat the procedure (update features in the parent node and check constraints) until the root segment or a node segment whose features don't need to be updated is reached. If this process terminates successfully then $s_{2}$ can continue $s_{1}$.

- if $8_{2}$ is to be replaced by a new segment $s_{3}$ then compute the features of segment $s_{3}$ and check if the set of temporal constraints of the old parent of segment $s_{1}$ is satisfied. If so, update the old parent $s_{1}$ features if needed and repeat the procedure (update features in the parent node and check constraints) until the root or a node segment whose features don't need to be updated is reached. If this process ends successfully then $s_{3}$ can replace $s_{1}$.

Whenever a temporal entity in the form of a discourse referent is added to the discourse structure, the structure containing all temporal discourse referents as well as their temporal constraints should be updated (fig. 4 for ex. of sec. 4). This way we can distinguish relations that are implied by the temporal system from those inferred using other knowledge sources. e.g. the constraints $t_{1}<t_{2}, t_{3}<t_{2}$ do not imply $t_{1}<t_{3}$ but satisfy it.

In order to check for temporal constraints we do not only deal with constraints over temporal intervals but use also world knowledge for abducting relations between eventualities that imply some temporal relation between them. In order to abduct a temporal relation it is not enough to block inconsistencies in the temporal system. There should also be some kind of justification, like:

- temporal - if the temporal system implies relation $t_{a_{1}} @ t_{s,}$ there is a justification to abduct $t_{t_{1}} @ t_{t_{2}}$, with@ being a temporal relation.

- causal - if $e_{1}$ can cause $e_{2}$ then there is a justification to abduct $t_{e_{1}}<t_{e_{2}}$.

- contingent - Assuming an event ontology like that of Moens and Steedman [17] where eventualities have a tripartite structure with a preparatory phase, a culmination and a consequence state there are clauses stating what are the eventualities of the preparatory phase and those of the consequence state of an eventuality.
- if $e_{1}$ can be in the preparatory phase of $e_{2}$ there is a justification to abduct $t_{e_{1}} \subset t_{e_{2}}$.

- if $e_{1}$ can be in the consequence state of $e_{2}$ there is a justification to abduct $t_{e_{1}}>t_{e_{3}}$.

- particular shared knowledge about eventualities - having a temporal knowledge base concerning eventualities, general rules for eventualities can be stated, e.g. John usually drinks a cup of coffee before he catches the bus.

- if $e_{2}$ usually happens $9 e_{1}$ with @ being a temporal relation, then there exists justification to assume $t_{e_{1}} @ t_{e_{2}}$.

- Linguistic - verb tense and aspectual perspective (AP) are the linguistic features that contribute to the temporal anchorage of eventualities. There are rules that justify a temporal relation taking into account these features and the order of the sentences. They should be used as default rules, i.e. if it can not be found another justification for a temporal anchoring then a linguistic justification should be used. The following rules are used in the detailed example.

if tense of $c_{1}$ and of $e_{2}$ is simple past (SP) with perfective AP then there is justification for assuming $t_{e_{1}}<t_{e_{2}}$.

- if tense of $e_{1}$ is SP with perfective AP and tense of $e_{2}$ is $\mathbf{S P}$ with imperfective $\mathbf{A P}$ there exists justification to assume $t_{e_{1}} \subset t_{e_{2}}$.

If a justification for the abduction of a particular temporal relation is not a logical consequence of the knowledge base then the justification should be added to the set of conditions. e.g. if we abduct in the discourse "John fell from the balcony. Mary pushed him." that John fell because Mary had pushed him, we should add the clause "cause $\left(e_{1}, e_{2}\right)$ " in order to block the future inference that Mary was innocent in John's fall. In this example another interpretation, linguistically justified, could be $t_{e_{\text {fall }}}<t_{e_{p u s h}}{ }^{6}$.

\section{Detailed Example}

Consider the following discourse represented by the segment in fig. 3 .

Last month I bought a house (s,). It had att aquarium $\left(s_{2}\right)$. Mary offered me a red fish $\left(s_{3}\right)$. John gave me his frog (a). My fish died yesterday $\left(\Delta_{5}\right)$. It stopped breathing ( $\left(_{0}\right)$. It became blue $\left(\Delta_{7}\right)$. It went to the top of the aquarium $\left(s_{B}\right)$.

\footnotetext{
SThese features may restrict the set of posaible temporal relations between two eventualities.

When there are more then one posaible temporal relation a system must chose one, but it should be able
} 


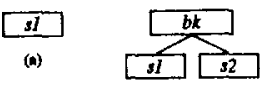

(b)

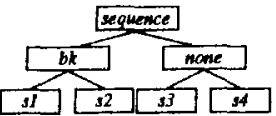

(d)

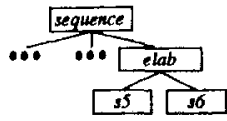

(n)

Figure 2: Segments while processing ex. sec. 4 .

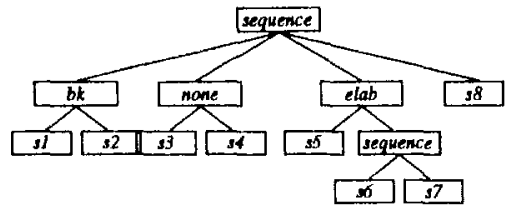

Figure 3: Segmentation for the ex. of sec. 4 .

Figure 2.a represents the discourse segment after processing sentence $s_{1}$. The only way to incorporate the sentence $s_{2}$ 's segment is by replacing the current discourse structure by a new one (basic segments cannot be continued). $s_{1}$ is the reference to anchor $s_{2}$, the eventuality of $s_{2}$ is a state, so the relation $t_{s_{1}} \subset t_{s_{2}}$ is abducted and the new segment is of sort $b k$ (fig. 2.b). To insert $s_{3}$ there are two visible segments: $s_{2}$ and $b k$. Segment $b k$ is the reference for $b_{3}$ because the eventuality of segment $s_{2}$ is a state with an imperfective aspectual perspective and there is no general knowledge about the eventualities of $s_{2}$ and $s_{3}$ allowing us to abduct a temporal relation between them. The features of the bk segment are the same as those of $s_{1}, 80$ the abducted relation between those segments is $t_{p_{1}}<t_{p_{1}}$, so that the discourse structure is replaced by a new segment of sort sequence (fig. 2.c). To insert segment $s_{4}$ there are two visible segments, namely $s_{3}$ and sequence. Using $s_{3}$ as reference, no temporal relation can be abducted. Thus none is abducted using some general rule saying "eventualities to give and to offer are of

to backtrack to that choice point.

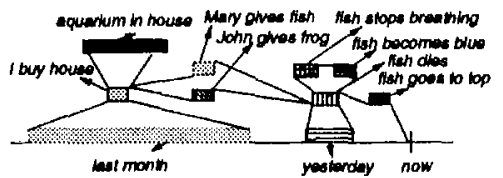

Figure 4: Temporal anchor of ex. sec. 4.

the same kind and if the subjects and objects are different and the recipient is the same it is not possible to abduct a temporal relation between them". So the segment $s_{3}$ is replaced by a new segment of sort none (fig. 2.d) after the check to see if it is possible to abduct the relation $t_{t_{k}}<t_{p_{1}}$ in order to satisfy the constraints of the sequence segment. The time of segment $s_{5}$ is partially anchored in the time interval "yesterday" but it still must be anchored to a reference. Visible segments are $s_{4}$, none and sequence. Considering that the abducted relation between $s_{4}$ and $s_{5}$ is $t_{9,}<t_{s_{s}}$ and between $s_{3}$ and $s_{5}$ is $t_{s,}<t_{s}$ it is not possible to use $s_{4}$ as referent because the constraints of segment none were not satisfied. So the sequence segment is the only one left and it can be continued by $s_{5}$ (fig. 2.e) because its constraints are satisfied, thus $t_{s,}<t_{s_{s}}, t_{p_{d}}<t_{s_{s}}$ are abducted. In order to insert $s_{6}$, the visible segments are $s_{5}$ and sequence. Using $s_{5}$ as referent, the relation $t_{s,} \subset t_{s s}$ is abducted because stop breathing is in the preparatory phase of dying. Segment $s_{5}$ is replaced by a new segment of sort elab (fig. 2.f). In order to insert segment $s_{7}$, segment $s_{6}$ is replaced by a new sequence segment because the relation $t_{0 s}<t_{s 7}$ is abducted (becoming blue is in the consequence state of stop breathing) and $t_{a}, \subset t_{a s}$ is abducted (becoming blue is in the preparatory phase of $d y i n g$ ) (fig. 2.g). The insertion of $s_{8}$ leads to the final structure (fig. 3). $s_{8}$ cannot use $s_{7}$ as reference because the relation $t_{\mathrm{s}} \subset t_{\mathrm{s} \mathrm{s}}$ cannot be abducted.

After processing the discourse, the temporal discourse referents and their relations reflect the structure of fig. 4, containing all the temporal relations that can be inferred from the text without making unjustified abductions.

\section{Conclusions}

Our segmentation method using abduction of temporal relations between eventualities as a 
leading criterion for segmentation ${ }^{\gamma}$ has advantages over other currently used segmentation techniques. It uses a criterion whose precision and feasibility is supported by previous work in the area of temporal anaphora resolution $[19,22,18,16,4]$. Our discourse segmentation provides the information necessary for tense interpretation (as required in [22]), and for constraining further segmentation. Although Webber's temporal focus structure depends on the linguistic discourse structure [7], she doesn't explain how tense interpretation contributes to the further development of the linguistic structure. Other work tackling the interpretation of tense and aspect $[19,18,13,4,2,16,3]$ does not intend to build up a discourse structure in the Grosz and Sidner's [7] sense. In [13] rhetorical relations between some eventualities are build up, not discourse segments. Work in discourse segmentation based on plan recognition [20] does not provide the necessary information for tense interpretation either. Our structure intends to provide the context necessary for the interpre tation of different kinds of anaphora. Our approach enables us to compute both, the temporal relations explicitly expressed in the discourse and those implied by the temporal structure of the discourse. The temporal coherence can be evaluated by looking at the final discourse structure, e.g. if a discourse is represented by a segment of sort none, no temporal relations between the eventualities described can be obtained and thus the discourse is not temporally coherent. Our approach does not prevent us from using complementary criteria for the further segmentation of discourse, namely from using other criteria such as clue words, spatial telations, and recognition of state elaboration.

\section{References}

[1] James Allen. Towards a General Theory of Action and Time. Artificial Intelligence, (23):123$154,1984$.

[2] K. Dahlgren and J. Mclowell. Knowledge representation for commonsence reasoning. Computational Linguistics, 15(3), September 1989.

[3] M. Dalrymple. 'The interpretation of tense and aspect in english. In Proc. $26^{\text {th }}$ An. Meet. of the ACL, June 1988 .

[4] K. Eberle and W. Kasper. Tense, aspect and temporal structure in French. In IL. Kamp, ed-

\footnotetext{
${ }^{7}$ In [8] abduction is also the lending process in
} int erpretation. itor, Tense and Aspect in English and French, Dyana deliverable R2.3.B, Jan 1991.

[5] K. Eberle and W. Kasper. Tenses as anaphora. In Proc, th Europ. Chap. of the ACL, 1989.

[6] F.v. Eynde. The semantic of tense and aspect. In M. Filgueiray et al, editor, NLP, FAIA' 9 , Springer-Verlag, Oct 1990.

[7] H. Grosz and C. Sidner. Attention, intention, and the structure of discourse. Computational Linguistics, $12(3), 1986$.

[8] J. Hobbs, M. Stickel, P. Martin, and D. Edwards. Interpretation as abduction. In Proc. 26th An Meet. of $A C I, 1988$.

[9] J. R. Hobbe. Coherence and coreference. Cognitive Science, $\mathbf{3}(1), 1979$.

[10] E. H. Hovy. Planning coherent multisentential text. In Proc. 26th An. Meet. of ACL, 1988.

[11] II. Kamp and C. Rohrer. Tense in texts. In C. Bauerle, R. Schwarze and A. von Stechow, editors, Use and Interpretation of Language, de Gruyter, 1983.

[12] K. Kowalski and M. Sergot. A Logic-based Calculus of events. New Generation Computing. (4):67-95, 1986 ,

[13] A. Lascarides and N. Asher. Discourse relations and defeasible knowledge. In Proc. 29th $A n$. Meet. of ACL, 1991.

[14] A. Lascarides and J. Oberlander. Temporal coherence and defeasible knowledge. In Workahop on Discourse Cohenence, Univ. of Edinburgh, April, 1991.

[15] W. Mann and S. Thompson. Rhetorical Structure Theory. Technical Report, Univ. of Southern Califoria, 1987.

[16] M. Moens. Tense Aspect and temporal refer. ence. Centre for Cognitive Science, Univ. of Edinburgh, 1987.

[17] M. Moens and M. Steedman. Iemporal ontology and temporal reference. Computational Linguistics, 14(2):15-28, 1988.

[18] A. Nakhimovsky. Aspect, aspectual class, and temporal structure of narrative. Computational Linguistics, 14(2):29-43, 1988.

[19] B. Partee. Nominal ant temporal anaphora. Linguistics and Philosophy, 7:243-286, 1984.

[20] L. Polasyi. A formal model of the structure of discourse. Journal of Pragmatics, 12:601-638, 1988.

[21] B. L. Webber. Discourse deixis: reference to discourse segments. In Proc. $26^{\text {th }}$ An. Meet. of A C L 1988.

[22] B. I. Webber. Tense as discourse anaphor. Computational Linguistics, 14(2):61-73, 1988. 